\title{
Functional micro/nanobubbles for ultrasound medicine and visualizable guidance
}

\author{
Chen Zhang ${ }^{1,2 \dagger}$, Yihong $\mathrm{Li}^{1,2 \dagger}$, Xinyong $\mathrm{Ma}^{3}$, Wenxin $\mathrm{He}^{1,2}$, Chenxi Liu ${ }^{1,2}$ \& Zhe Liu ${ }^{1,2 \dagger^{*}}$ \\ ${ }^{1}$ Academy of Medical Engineering and Translational Medicine, Tianjin University, Tianjin 300072, China; \\ ${ }^{2}$ Tianjin Key Laboratory of Brain Science and Neural Engineering, Tianjin University, Tianjin 300072, China; \\ ${ }^{3}$ Division of Academic \& Cultural Activities, Academic Divisions of the Chinese Academy of Sciences, Beijing 100190, China
}

Received December 13, 2020; accepted January 18, 2021; published online February 25, 2021

\begin{abstract}
Chemically functionalized gas-filled bubbles with a versatile micro/nano-sized scale have witnessed a long history of developments and emerging applications in disease diagnosis and treatments. In combination with ultrasound and image-guidance, micro/nanobubbles have been endowed with the capabilities of biomedical imaging, drug delivery, gene transfection and diseaseoriented therapy. As an external stimulus, ultrasound (US)-mediated targeting treatments have been achieving unprecedented efficiency. Nowadays, US is playing a crucial role in visualizing biological/pathological changes in lives as a reliable imaging technique and a powerful therapeutic tool. This review retrospects the history of ultrasound, the chemistry of functionalized agents and summarizes recent advancements of functional micro/nanobubbles as US contrast agents in preclinical and transclinical research. Latest ultrasound-based treatment modalities in association with functional micro/nanobubbles have been highlighted as their great potentials for disease precision therapy. It is believed that these state-of-the-art micro/nanobubbles will become a booster for ultrasound medicine and visualizable guidance to serve future human healthcare in a more comprehensive and practical manner.
\end{abstract}

ultrasound, functional biomaterials, image guidance, visualizable medicine, drug delivery

Citation: Zhang C, Li Y, Ma X, He W, Liu C, Liu Z. Functional micro/nanobubbles for ultrasound medicine and visualizable guidance. Sci China Chem, 2021, 64: 899-914, https://doi.org/10.1007/s11426-020-9945-4

\section{Introduction}

\subsection{Nature and application scope of ultrasound}

The commonly used techniques presently for disease diagnosis and treatments include molecular imaging, gene tests, biomarker analysis, endoscopy, surgery, chemotherapy and radiotherapy. Among them, ultrasound imaging has been widely used in ophthalmology, gynecology and obstetrics, cardiovascular, digestive and urinary systems. Ultrasound (US) is defined by the American National Standards Institute (ANSI) as a type of acoustic waves with a frequency higher

$\dagger$ These authors contributed equally to this work.

*Corresponding author (email: zheliu@tju.edu.cn) than $20 \mathrm{kHz}$, the upper audible frequency limit by humans $[1,2]$. Sent out by an ultrasound transducer and backscattered by different organs and tissues, the acoustical waves can be recorded and reconstructed into a US image by an imaging acquisition system. The ultrasound can not only be utilized for diagnostic imaging, but also help alleviate joints and muscles pains. Additionally, with the aid of ultrasound contrast agents (US CAs), image contrast can be significantly enhanced, and US-induced cavitation and sonoporation will present a good avenue for boarder biomedical applications (Figure 1) [3]. It has become one of the most commonly used and powerful imaging techniques due to its multitude of prominent attributes, such as non-invasiveness, ionization-free radiation, real-time, low cost, great spatial 


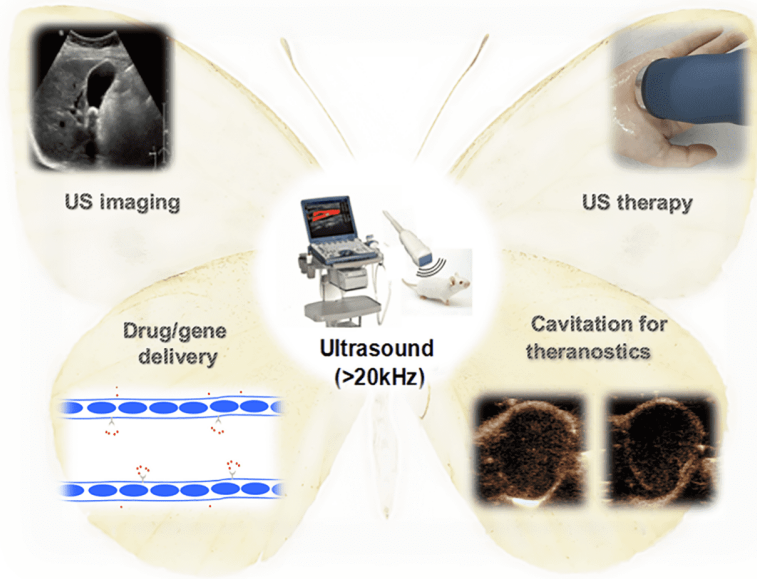

Figure 1 A schematic image of ultrasound biomedical applications, e.g. diagnostic imaging for fetal anomalies and therapy for injured muscles/ joints. In combination with US CAs, it helps deliver drug/genes as well as precision medicine, and takes advantage of their unique cavitation and sonoporation phenomena through effective localization (color online).

and temporal sensitivity, and capability to acquire structural and functional images simultaneously [4-6].

Meanwhile, an evident growth in ultrasound research was observed based on 1999-2019 PubMed statistics. The publications interpreted by emergency, surgery, oncology, radi- ology, preclinical and US CAs have increased by $731 \%$, $297 \%, 300 \%, 202 \%, 162 \%$, and $172 \%$ respectively (Figure 2). Moreover, ultrasound devices nowadays displayed portable and readily access to public uses [7].

\subsection{Functional micro/nanobubbles}

In the past years, US CAs have witnessed roaring developments in not only disease diagnosis but also biomedical treatment. The earliest study on US CAs dated back to 1968, when Gramiak [8] found that free air-bubbles significantly increased the backscattered echoes during echocardiographic examinations, but those first saline bubbles were unstable due to the high surface tension. In 1970, Kremkau [9] confirmed that the intracardiac injection of autologous blood at rapid rates could produce numerous small gas bubbles and enhance significant contrast echoes. Nevertheless, there was still a pressing need for prolonging bubbles lifetime and defining a narrowly dispersed size. Since then, plenty of efforts were dedicated to improve the stability and biocompatibility. In 1990, the first US CAs (Albunex), an albumin-stabilized and air-filled microbubble was approved by Food and Drug Administration (FDA) and its commercial availability was announced [10].
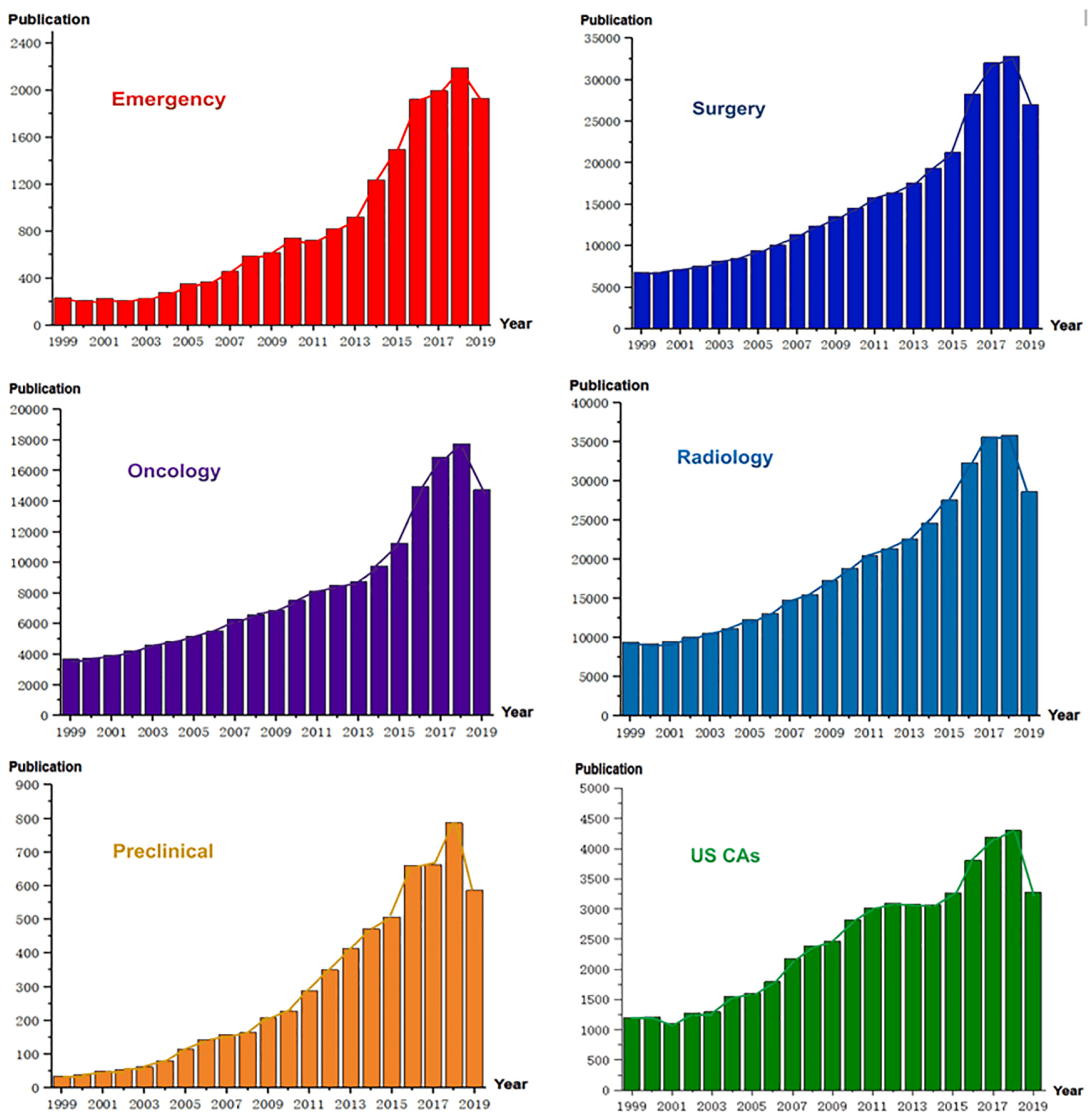

Figure 2 A roaring increase of ultrasound publications and applications in the past two decades from 1999 to 2019 , interpreted by emergency, surgery, oncology, radiology, preclinical research and US CAs respectively (color online). 
One particular advantage of microbubbles is that they are highly stable and are manufactured at a relatively low cost. With the development of materials science, different kinds of commercial US CAs with versatile shell compositions and gas cores have already been applied as diagnostic agents in the past decade as listed in Table 1 [11]. Those approved US CAs are commonly used in the clinics, contrast enhanced ultrasound (CEUS) imaging of the liver tissue guarantees proper and comparable examinations for the patients' diagnosis and therapy. In addition, ultrasound is combined with varied medical imaging modalities, such as magnetic resonance imaging (MRI) and/or optical imaging (OI), to achieve more accurate visualizable guidance. Multi-modal image-guided measures provide a more effective approach for the diagnosis and therapy of cancers.

\section{Chemistry of various micro/nanobubbles for US-mediated theranostics}

\subsection{Microbubbles}

Microbubbles (MBs) are gas-liquid emulsions, consisting of a gaseous core surrounded by a shell of biocompatible properties (e.g., made of albumin, lipids, protein or polymer) and usually of a diameter between 1-10 $\mu \mathrm{m}$. MBs have a protective shell structure to avoid gas leakage and particle fusion. MBs are composed of different shell compositions and a chemically-inert gas core. Normally, the mechanical elasticity of MB depends on the material of the shell, and the more elastic the material is, the stronger acoustic energy microbubbles can tolerate.

Once applied to US, MBs give rise to acoustic impedance mismatch between blood and tissues, resulting in a dramatic enhancement of ultrasonic backscattered signals with an excellent signal-to-noise ratio (SNR). Due to the material characteristics, the shell material provides multiple possibilities for tailoring MBs to their specific application by changing visco-elastic properties. Normally, shell materials are classified as soft- and hard-shell according to their viscoelastic properties.

\subsubsection{Hard-shelled microbubbles}

Hard-shelled MBs are composed of a gas core with coating materials (such as polymers or denatured proteins) whose visco-elastic property is relatively lower. Generally, hardshelled MBs are stable even under higher-intensity US irradiation and show a longer circulation time in vivo.

Protein shells

Albumin-shelled MBs were pioneered contrast agents used in CE-US imaging [18]. These MBs can pass the lung capillaries and then provide high contrast in the left ventricle of heart. Albunex is the first FDA-approved albumin-shelled microbubbles with a diameter from 1 to $15 \mu \mathrm{m}$, and the shell stabilization is assured by disulfide bonds between cysteines. To develop albumin-coated microbubbles, Keller et al. [19] sonicated heat-denatured 5\% (w/v) human albumin solution in the air atmosphere. During sonication, air-filled MBs were formed with a diameter of approximately $10 \mu \mathrm{m}$ and a shell thickness of $15 \mathrm{~nm}$. Further studies confirmed that intracoronary injection of these MBs did not alter coronary blood flow and no significant hemodynamic effect was detected.

Apart from Albunex, the second-generation albumin formulation of Optison was developed by using perfluorocarbon as the gas core. The size was about 2-5 $\mu \mathrm{m}$ and the shell thickness was approximately $15 \mathrm{~nm}$. Clinical trials have shown that Optison has a relatively prolonged and a better contrast enhancement compared to Albunex [20]. Moreover, the low solubility of perfluorocarbon results in a much longer circulation lifetime.

In addition to albumin, Cavalieri [21] synthesized functional MBs coated with chemically reduced lysozyme by mean of high-intensity US in aqueous solution. The lysozyme-coated MBs were stable for months and the enzymatic (antimicrobial) activity of lysozyme was maintained.

\section{Polymer shells}

Polymer shelled MBs refer to a special class of MBs that are stabilized by a thick polymeric shell. Compared to the commercially available lipid shelled MBs, it's more favorable to use polymer shelled CAs with their unique advantages. The most important one is that the polymer shell is more robust to mechanical stress than lipids [22]. In 1990, Wheatley [23] described a new kind of polymer based US CAs, whose shell was made up of alginate. The MBs were formed by concentric jets of air and alginate solution that were sprayed into a reservoir. The alginate was adsorbed to

Table 1 Representative commercial ultrasound contrast agents for clinical uses

\begin{tabular}{|c|c|c|c|c|c|}
\hline Contrast Agent & Manufacturer & Shell & Gas & Size $(\mu \mathrm{m})$ & Application \\
\hline Albunex [12] & Molecular Biosystems & Albumin & Air & 4.3 & Cardiovascular imaging \\
\hline Optison [13] & GE Healthcare/Amersham & Albumin & $\mathrm{C}_{3} \mathrm{~F}_{8}$ & $2.0-4.5$ & Cardiovascular imaging \\
\hline Definity/ Luminity [14] & LantheusMedical Imaging & Lipid/ Surfactant & $\mathrm{C}_{3} \mathrm{~F}_{8}$ & $1.1-3.3$ & Cardiovascular imaging \\
\hline Sonovue [15] & Bracco Diagnostics & Lipid & $\mathrm{SF}_{6}$ & $2.0-3.0$ & Carotid plaque imaging \\
\hline Sonazoid [16] & GE Healthcare/Amersham & Lipid & $\mathrm{C}_{4} \mathrm{~F}_{10}$ & 2.6 & Tumor imaging \\
\hline Levovist [17] & Shering AG & Lipid/Galactose & Air & $2.0-4.0$ & Carotid plaque imaging \\
\hline
\end{tabular}


the gas/liquid interface and was hardened upon plunging into the calcium solution. Their size could be controlled by adjusting the flow rates of air around the syringe needle.

With the development of polymer science, a number of polymer-shelled MBs were fabricated by different membrane materials and core gases. Among them, poly D,Llactide-co-glycolide acid (PLGA) is a commonly used material for MBs synthesis. In 1999, Nayaran and Wheatley [24] firstly reported the hollow microcapsules formed by the biodegradable copolymer of PLGA. PLGA-coated microcapsules were prepared by an adaptation of either a solvent evaporation or a coacervation method. After intravenous injection, these MBs showed a significant contrast enhancement inside the rabbit kidney in color Doppler imaging. Since then, PLGA-based MBs were developed and widely utilized as both US CAs and functional carriers for drug/gene delivery [25-27].

Cyanoacrylates are commonly used shell materials for MBs synthesis. Bauer $[28,29]$ evaluated the imaging properties and diagnostic potential of US CAs SHU 563A. The microspheres of SHU 563A remained intact while circulating in the blood pool for up to $10 \mathrm{~min}$ after intravenous injection. Followed by that, some other cyanoacrylate polymers are employed as well. For instance, poly butyl cyanoacrylate (PBCA) is a well-known biocompatible polymer which was reported as an excellent candidate for preclinical CE-US imaging and cancer diagnosis [30]. In addition to US imaging, the surface of the PBCA MBs could be easily functionalized by conjugating antibodies/peptides to improve their specific cell targeting capability. Meanwhile, the incorporation of other functional nanoparticles into the shells could change PBCA MBs into a fascinating multi-modal US CAs [31,32].

Cavalieri [33] firstly described the synthesis of poly (vinyl alcohol) (PVA) based MBs, which were created by chemical cross-linking at the air-water interface of an acidic solution of telechelic PVA via acetalization ultrahigh-speed stirring, and the average diameter of MBs could be controlled by changing reaction temperature. The major advantage of PVA MBs was their long shelf-life of several months. Moreover, incorporating superparamagnetic iron oxide nanoparticles (SPIONs) into the shell could realize multi-modal imaging [34]. The magnetic properties of the nanoparticles contributed to a strong spin-spin relaxation time effect, making them suitable as enhancers for MRI. Therefore, they could be used in diagnostics imaging including US and MRI.

Bohmer [35] reported the fabrication of copolymer polyperfluorooctyloxycaronyl-poly(lactic acid) (PLA-PFO) shelled, narrowly size-distributed MBs by ink-jet printing technology. The pressure generated by the piezoelectric crystal led to the formation of a droplet at the nozzle. The capsule size could be readily controlled by varying the frequency and length of the pressure pulses.

\subsubsection{Soft-shelled microbubbles}

Soft-shelled MBs display a high degree of sensitivity when exposed to pressure change. This property of such MBs can be ascribed to no coating or a monolayer coating with surfactant molecules such as palmitic acid or phospholipids. Due to their great compressibility, soft-shelled MBs have high echogenicity. Moreover, the major advantage of this kind of lipid-shelled material is their ability to spontaneously assemble in mono-, bi-, or multi-layers forming micelles or liposomes. However, lack of sufficient stability especially in time-consuming ultrasound scans makes them highly interesting compounds for CE-US imaging. To overcome this disadvantage of soft-shelled MBs, the introduction of polyethylene-glycol (PEG) to the shell is conducive to extend their life-span.

Lipid shells

Lipid-coated MBs are one of the most interesting and useful formulations due to plenty of advantages. Phospholipids spontaneously self-assemble into a highly oriented monolayer at the air-water interface, with the hydrophobic acyl chains facing the gas and the hydrophilic head groups facing the water. Perflutren is the first lipid-based US CAs used for clinical trials, and is approved as an i.v. injectable US CAs. Sold as Definity or Luminity, they are composed of perfluoropropane gas core and coated with saturated $\mathrm{C}_{16}$ long phospholipids dipalmitoylphosphatidylcholine (DPPC), dipalmitoylphosphatidic acid (DPPA) and dipalmitolyphosphatidylethanolamine- PEG $_{5000}\left(\mathrm{DPPE}-\mathrm{PEG}_{5000}\right)$ [36]. The mean size is approximately $1-2 \mu \mathrm{m}$ in diameter and about $99 \%$ are less than $7 \mu \mathrm{m}$. They do not aggregate on storage and appear as a milky white color suspension. Also, lipidshelled MBs are highly echogenic because of their flexibility, which allows them to resonate under ultrasound stimulation with minimal damping. Although Perflutren is approved for cardiovascular diagnosis, Sanchez [37] further expanded their applications in the diagnosis of renal cell carcinoma.

Sonovue is the best illustrated commercial US CAs for cardiologic applications, which is comprised of insoluble gas and a phospholipid monolayer. Sulfur hexafluoride $\left(\mathrm{SF}_{6}\right)$ and perfluorocarbon gases, such as perfluorobutane $\left(\mathrm{C}_{4} \mathrm{~F}_{10}\right)$ and perfluoropropane $\left(\mathrm{C}_{3} \mathrm{~F}_{8}\right)$, are appropriate candidates due to their inert and non-toxic property and low solubility in aqueous medium. Stabilization of microbubbles by lipid coatings could not only increase the plasma half time, but also narrow their size distribution and preserve their physical integrity.

This kind of $\mathrm{SF}_{6}$-filled phospholipid MBs are commonly used for left ventricular opacification and endocardial border imaging. Recently, some countries approved them for general vessel diagnostic imaging of microvascular structures in the breast or differentiation of lesions in the liver [11]. Apart from that, Sonovue has also been tested in clinical trials for monitoring of uterine fibroid vascularization and improved 
ablation [38].

Surfactant shells

In 1993, Singhal [39] firstly reported the production of stable surfactant MBs by sonicating the mixtures of two nonionic surfactants, $\operatorname{Span}_{60}$ and $\mathrm{Tween}_{80}$ at different molar ratios. Results showed that there was the highest yield of MBs at a molar ratio of $\operatorname{Span}_{60}: \mathrm{Tween}_{80}$ of 0.63 . Around $80 \%$ of the MBs possessed a particle size less than $8 \mu \mathrm{m}$, and $76 \%$ of MBs' diameters were between 4 and $10 \mu \mathrm{m}$, an excellent size distribution for CAs.

Imagent is a perfluorohexane-stabilized US CAs in the assessment of cardiac function and myocardial perfusion, as well as the detection of solid organ lesions and blood flow abnormalities caused by vascular diseases and malignancies. In the preclinical studies, it has been found to enhance the signal from perfused tissues and blood vessels through using gray-scale, color Doppler and harmonic ultrasound imaging techniques. More impressively, they were firstly tested for renal and liver perfusion studies in rabbits. It showed a promising contrast and compatibility with almost no adverse effects in clinical trials $[40,41]$.

In the following years, a great deal of surfactant-shelled MBs have been developed and applied in both research and clinics. Levovist was developed in the late 1980s and since then, it was tested and used for several clinical diagnostic applications. The CA was consisted of a saccharide- and palmitic acid-containing shell and air, and clinical trials in patients showed obvious contrast enhancement. Ota and $\mathrm{Wu}$ $[42,43]$ further expanded the application area using Levovist for differentiating benign and malignant tumors in several organs, such as spleen and ovaries. Their results showed that it was possible to tell the difference between malignant and benign tumors so as to avoid unnecessary biopsies and surgeries.

\subsection{Nanobubbles}

Ultrasound in combination with MBs presents a good avenue for not only diagnostic applications but also US-mediated therapeutics to various lesions. However, the intrinsic large size limits its further development. Conventional microsized US CAs injected parenterally are less than $8 \mu \mathrm{m}$ which could traverse the capillaries in the pulmonary bed, but still remain in the vasculature until eliminated from the body [44]. Also, the large size led them unable to target the specific tissues. For example, tumors are characterized by a leaky vasculature with large gaps between the endothelial cells, allowing the access of nanoparticles less than $700 \mathrm{~nm}$ [45]. Taken fast clearance of MBs by the reticuloendothelial system and the inability to penetrate the tumor fenestrate openings into consideration, there is an urgent need to reduce the US CAs size for broader theranostic applications.

Researchers have made a consistent effort to find a compromise between particle size and echo intensity. There has been insurmountable hurdle to deal with the fact that the high echo signal will be offset due to the reduction of the particle size. In detail, MBs show strong echo signals for US imaging. However, even though nano-sized particles are capable of penetrating into specific tissue, they cannot exhibit echo intensity enough for imaging (Figure 3) [46].

Similar to MBs, nanoparticles embedded with intact gas core, namely nanobubbles (NBs), have been developed as a promising agent for imaging and/or therapeutic use. NBs comprised of a gas core and different shell materials (polymers, phospholipids and so on) show great enhancement in US imaging $[44,47]$.

Yin [48] fabricated a phospholipid-shelled gas-core nanobubble and verified its in vivo contrast enhanced imaging of tumor. The NBs were comprised of octafluoropropane gas core and lipid shell with small, spherical, non-aggregating particles. The average diameter was $436.8 \pm 5.7 \mathrm{~nm}$ compared with $1220 \pm 65 \mathrm{~nm}$ of the control MBs. The enhancement lasted for relatively longer periods (approx. $1 \mathrm{~h}$ ) while the enhanced signal of control MBs lasted only for $15 \mathrm{~min}$. This phenomenon could be ascribed to the enhanced permeability and retention (EPR) effect of nano-sized materials, by which NBs passed through the pores in tumor vasculature then accumulated and retained in tumor for a much longer time.

Besides NBs with polymeric shells have remarkably enhanced US contrast. Xu [45] developed NBs with PLGA shell surrounding an air core with fluorescent dye pf Texas Red encapsulated. The average size of NBs was $268 \mathrm{~nm}$, and tumor targeting ligand HuCC49 $\triangle \mathrm{CH} 2$ antibody was introduced for the specific targeting of TAG-72, which is overexpressed in many epithelial-derived cancers. Furthermore, Texas Red together with NBs enabled the fluorescent/
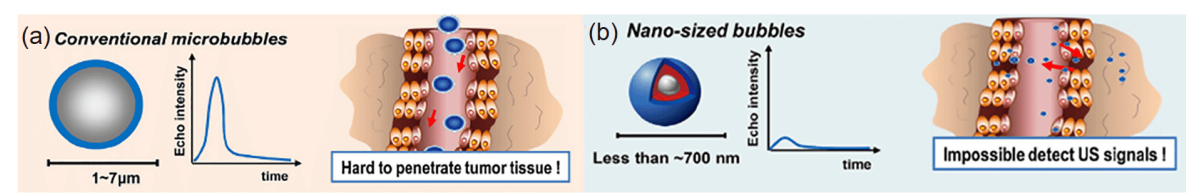

Figure 3 Schematic illustrations of microbubbles and nano-sized bubbles differences. (a) Conventional microbubbles possess high echo intensity but very short echo persistence and half-life time inside the body. Meantime, their extravasation from vessel to surrounding tissue is limited by their large size. By contrast, (b) nano-sized bubbles show the desirable distribution and great targeting ability but the echo signals generated is not enough for imaging (Reproduced from Ref. [46] with the permission of Elsevier Ltd.) (color online). 
US imaging, and the dual-modal imaging was demonstrated with LS174T human colon cancer cells.

\section{US-based diagnostic evaluations with visua- lizable guidance}

\subsection{Evaluations by US imaging}

US Imaging is a non-invasive technique that uses sound energy to visualize tissue and organs in the body. US imaging currently has widespread used in the clinical setting for numerous applications ranging from body imaging, echocardiography (cardiac imaging), vascular imaging, to intravascular imaging of the blood vessel wall. US imaging often uses CAs to illuminate tissues and vessels which may be of particular interest to the physicians.

MBs have long been thought to be the excellent CAs for US imaging. Hundley [49] injected MBs based CAs intravenously to evaluate whether contrast-enhanced transthoracic echocardiography improves the evaluation of left ventricular (LV) volumes and ejection fraction (EF), and the results confirmed that the enhanced contrast improved the visualization of the endocardial border and thereby increased the accuracy of echocardiography in assessing LV size and systolic performance at rest or during stress.

Rainbird [50] compared non-contrast imaging with contrast imaging of the LV during dopamine stress echocardiography (DSE) by intravenous injecting Optison. The results demonstrated that both the wall segment visualization and image quality at rest or at peak stress were improved by using Optison, resulting in improved confidence of interpretation.

Contrast enhancement during echocardiography can also be useful for identifying the presence of mural thrombus, left ventricular pseudoaneurysms and aortic dissection. Kono [51] evaluated B-mode US angiography enhanced with FS069 (a US contrast agent approved by FDA for endocardial border delineation) in human subjects with carotid artery disease. These findings confirmed that contrast-enhanced gray-scale US with phase-inversion harmonic imaging filled the carotid arteries with echoes and highlights plaques in human subjects. They could be used to improve the accuracy of Doppler-based methods for measuring intracardiac or intravascular flow velocities that are used to evaluate haemodynamic abnormalities.

Besides that, MBs could also be used to detect abnormal flow patterns or anatomy. Darge [52] performed a diagnostic examination of vesicoureteral reflux undergoing standard sonography of the urinary tract in the fundamental mode, followed by intravesical administration of a galactose-based contrast medium containing MBs. Visualization of the urinary tract and detection of ultrasound contrast media were significantly improved by the use of the harmonic imaging modalities. Moreover, the real-time monitoring of in vivo
US-mediated drug delivery can be realized via MRI/US or fluorescence/US dual-modal imaging system.

\subsection{Evaluations by multi-modal imaging}

A number of imaging platforms are available for molecular imaging, including optical imaging, positron-photon emission tomography or single-photon emission computed tomography (PET or SPECT), MRI and CEUS. US remains the most widespread technique for diagnostic imaging. These imaging capabilities provide the basis for in vivo, multimodal, and targeted molecular imaging [53]. Each imaging platform is dependent on specific imaging moieties, such as superparamagnetic iron oxide nanoparticles (SPIONs), ${ }^{99 \mathrm{~m}} \mathrm{Tc},{ }^{18} \mathrm{~F}$, or MBs. Molecular imaging enables the study of biological processes such as inflammation, thrombosis, phagocytic activity, metabolic activity, lipid uptake, oxidative stress, proteinase activity, neoangiogenesis, osteogenesis and thrombosis, each with specific molecular targets [54].

\subsubsection{US-MRI combination}

MRI and US imaging are widely used in diagnosis and therapy. One of their synergies, MRI-guided focused ultrasound therapy, is a noninvasive thermal ablation method approved by FDA for the cure of uterine fibroids. It is also developed as a treatment for liver, bone, prostate and brainrelated diseases $[55,56]$.

MRI-detectable MBs are considered to be a fascinating idea since it can be used not only to image cavitation events, but also to determine the distribution of MBs' shell debris.

Our group has developed an ultrasmall superparamagnetic iron oxide (USPIO) nanoparticles-embedded polymeric MBs [31]. These hybrid CAs undoubtedly have advantages such as strong contrast in US, and increased transversal relaxation rate in MRI. Once applied to external ultrasound field, a significant increase in relaxivities could be observed due to the US-induced bubble destruction. This phenomena was ascribed to the greater interaction of the released iron oxide nanoparticles with surrounding water molecules (Figure 4). The relaxivities were determined to investigate whether the local disintegration and the release of embedded USPIO can also be monitored by MRI.

The controllable opening of blood-brain-barrier (BBB) and drug delivery across BBB are playing an important role in the diagnosis and therapy against brain diseases and malfunctions and other central nervous system (CNS). In combination with MRI, fluorescence and US, PBCA-based microbubbles could be utilized to mediate and monitor the BBB opening process as well as depositing the ultrasmall USPIO nanoparticles into the brain [57]. The pulsed US exposure triggered the destruction of USPIO-embedded MBs and the encapsulated USPIO nanoparticles were released. Moreover, the vessel permeability was transiently enhanced 
(A)

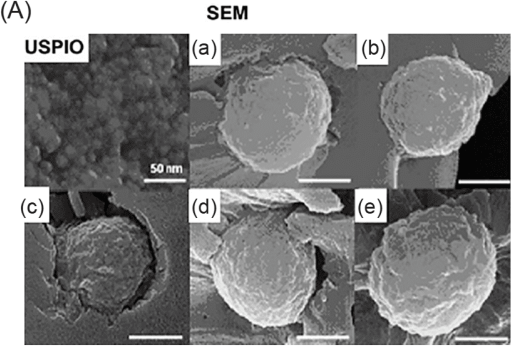

(C)

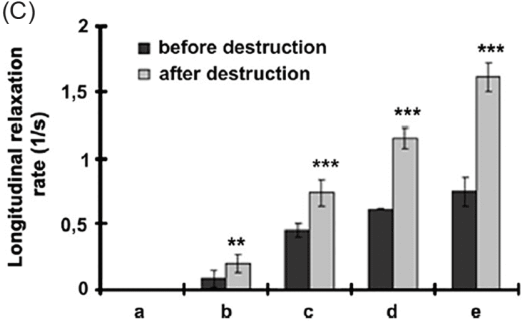

(B)

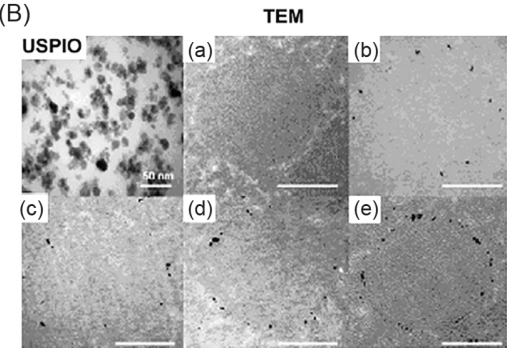

(D)

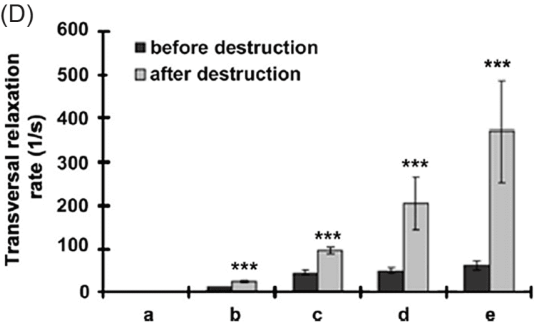

Figure 4 (A) SEM and (B) TEM images of USPIO nanoparticels, pure polymeric microbubbles (a) and USPIO embedded microbubbles with increasing iron concentration (b-e). Scale bar: $50 \mathrm{~nm}$ (USPIO); $1 \mu \mathrm{m}$ (panels a-e); (C) Longitudinal and (D) transversal relaxation rates of pure (a) and USPIOcontaining bubbles before and after US-induced MB destruction (Reproduced from Ref. [32] with the permission of Elsevier Ltd.).

by ultrasonic forces to render the extravasation of USPIO into the brain tissues. $\mathrm{R}_{2}{ }^{*}$-mapping and $\mathrm{T}_{2}{ }^{*}$-weighted imaging analysis also confirmed that the USPIO nanoparticles non-invasively extravasated from the vessels across the BBB and were successfully delivered into the brain.

Feshitan reported a lipid-coated microbubbles with the paramagnetic MRI contrast agent Gd(III)-DOTA chelation on the surface. Fragmentation of these intact Gd(III)-bound microbubble led to a remarkable increase in longitudinal molar relaxivity $r_{1}(40$-fold). This phenomenon could be explained as follows. Gd(III) was a paramagnetic ion that will show a prominent relaxivity when it interacted and exchanged with the surrounding water protons via its inner core [58]. Because the intact MBs were made up of a highly condensed monolayer shell, which might have restricted access of aqueous protons to the $\mathrm{Gd}(\mathrm{III})$ ion. Once destructed, the lipid shell would convert to a more incompact liposomal bilayer configuration, providing sufficient exchange with water molecules, hence giving rise to a great relaxation enhancement [59]. Further experiments supported this explanation by comparing the relaxivities change of MBs with Gd(III) bounded to two different locations on the lipid monolayer shell (the lipid headgroup region or the distal region of PEG brush) after US destruction. The result demonstrated that the significant $r_{1}$ increase of Gd(III)-headgroup bounded MBs and the slightly $r_{1}$ decrease of Gd(III)PEG bounded MBs after fragmentation further supported this porton-exchange enhancement mechanism [60].

\subsubsection{US-OI combination}

Ultrasound has been thought to be a major contributor towards anti-cancer drug delivery to tumor cells since the cell membrane permeability was significantly enhanced by US. However, monitoring of US-induced drug delivery to verify the underlying mechanism is still a vacancy. In the virtue of the prominent properties as high sensitivity, resolution ratio and easy access, fluorescence becomes the optimal choice to monitor the real-time US-mediated drug delivery in vivo.

Our group developed a synthetic protocol for PBCA-based MBs with intrinsic fluorescence and applied them to diagnostic applications of cell labeling and imaging (Figure 5) [61]. Common fluorescent dyes or therapeutic drugs such as Rhodamine-B, Coumarin- 6 , Nile Red and DOX could be encapsulated inside the MBs. With subcutaneous injection of these bubbles, contrasts of fluorescent signals could be detected before, during or after the injection processes, showing sufficient sensitivity to allow dynamic monitoring in small animals.

In addition to the encapsulation of fluorescent agents inside MBs, we developed genipin cross-linked REDV-conjugated polymeric MBs for human vascular endothelial cell (HVEC) labeling and targeting [62]. The cross-linking between genipin and chitosan not only offered the generation of intrinsic fluorescence, but also gave rise to the potentially enhanced loading capacity of drugs/genes (Figure 6(a)). The low in-vitro cytotoxicity and biodegradable profiles have also been validated considering their availability as carriers for drug delivery/gene transfection (Figure 6(b)). Furthermore, the active targeting assay disclosed that the binding process between REDV-conjugated MBs and endothelial cells could be readily visualized and evaluated under confocal laser scanning microscopy (CLSM) (Figure 6(c)). Based on these findings, this kind of MBs have become a powerful theranostic platform for ultrasound-triggered drug 


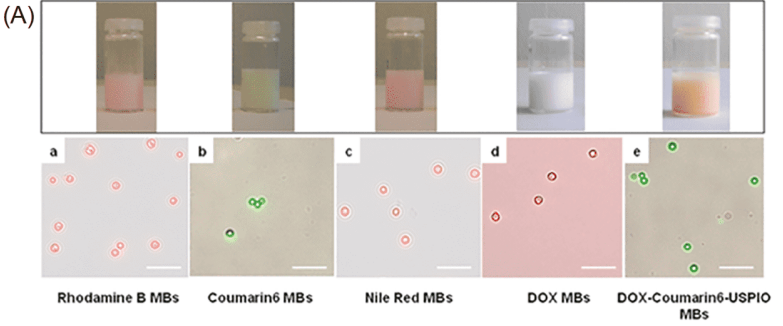

(B)

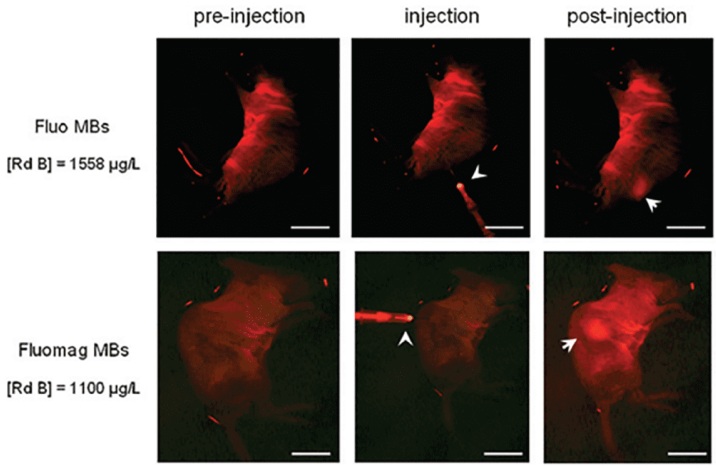

Figure 5 Various PBCA-based fluorescent MBs (A) for fluorescence image-guidance with subcutaneous injection (B) (Reproduced from Ref. [61] with the permission of Royal Society of Chemistry Ltd.) (color online).

release or ultrasound-mediated gene therapy with high precision and efficiency for a variety of vascular diseases.

Multidrug resistance (MDR) is still one of the main obstacles in colorectal cancer chemotherapy. Dai [63] proposed an efficient combination strategy based on porphyrin/ camptothecin-floxuridine microbubbles (PCF-MBs) with high drug loading contents, which showed high co-delivery stability and no premature release. The PCF-MBs acted as both US CAs and fluorescence imaging probes which also played a synergistic chemo-photodynamic therapy overcoming the MDR effects (Figure 7).

David reported US-triggered conversion of high payload porphyrin-encapsulated MBs to NBs, which actively enhanced cell permeability for local drug delivery. The cell permeability could be visualized real-time by the integrated acoustic and microscopic setup. As compared with the controls, the extravascular fluorescence in treated mice increased to 5-fold higher within 15 mins and dropped to less than 1.3 fold over a longer time-span of 65 mins due to the passive leakage [64].

\subsubsection{US-PAI combination}

Photoacoustic imaging (PAI) has become an emerging diagnostic tool nowadays and has found vast applications in photoacoustic microscopy (PAM), photoacoustic endoscopy (PAE) and whole-body photoacoustic imaging [65]. As an integrated biomedical imaging modality, it combines the advantages of acoustic deep penetration and optical high sensitivity, providing the functional and structural images with satisfactory resolution and contrast. Thus, abundant valuable biological or pathological information can be assessed for disease-oriented diagnosis and treatment by using PAI, and both endogenous and exogenous photo-absorbers can be used as PAI contrast agents to significantly enhance the sensitivity and the theranostic efficiency [66]. (a)

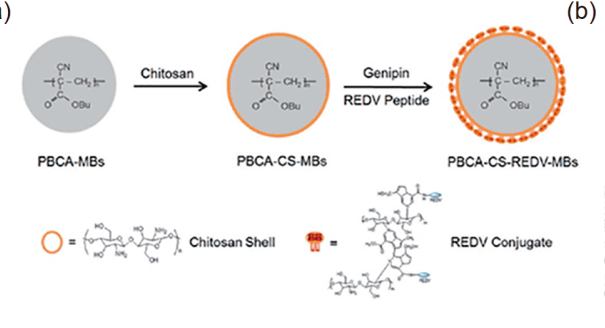

(c) 1)

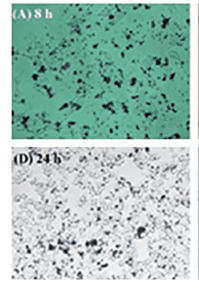

2)

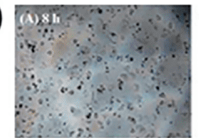

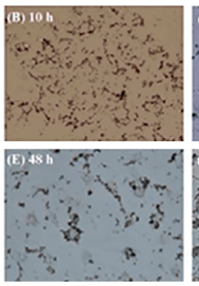
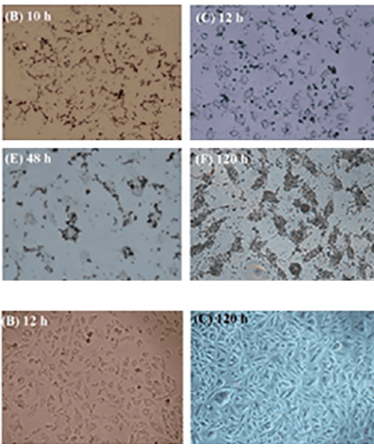

(b)
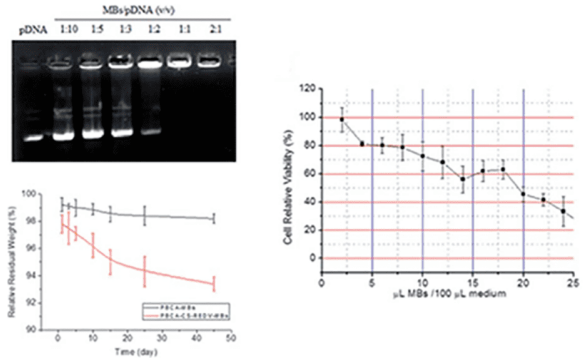

3)

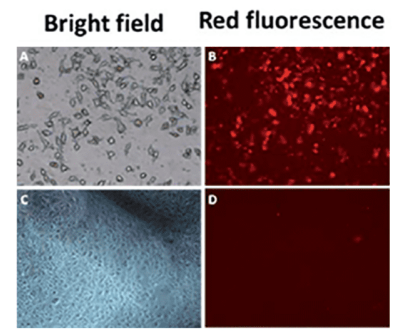

Figure 6 Fluorescent genipin cross-linked REDV-conjugated polymeric MBs for HVEC targeting. (a) The fabrication protocol via chitosan surface-coating, genipin cross-linking and REDV peptide conjugation; (b) agarose gel electrophoresis of MBs/pEGFP complexes, in-vitro biodegrability and cytotoxicity profiles; (c) active binding performance of REDV-conjugated MBs to endothelial cells (ECs) in comparison to smooth muscle cells (SMCs) at different time points under the visualization of CLSM (Reproduced from Ref. [62] with the permission of Royal Society of Chemistry Ltd.) (color online). 

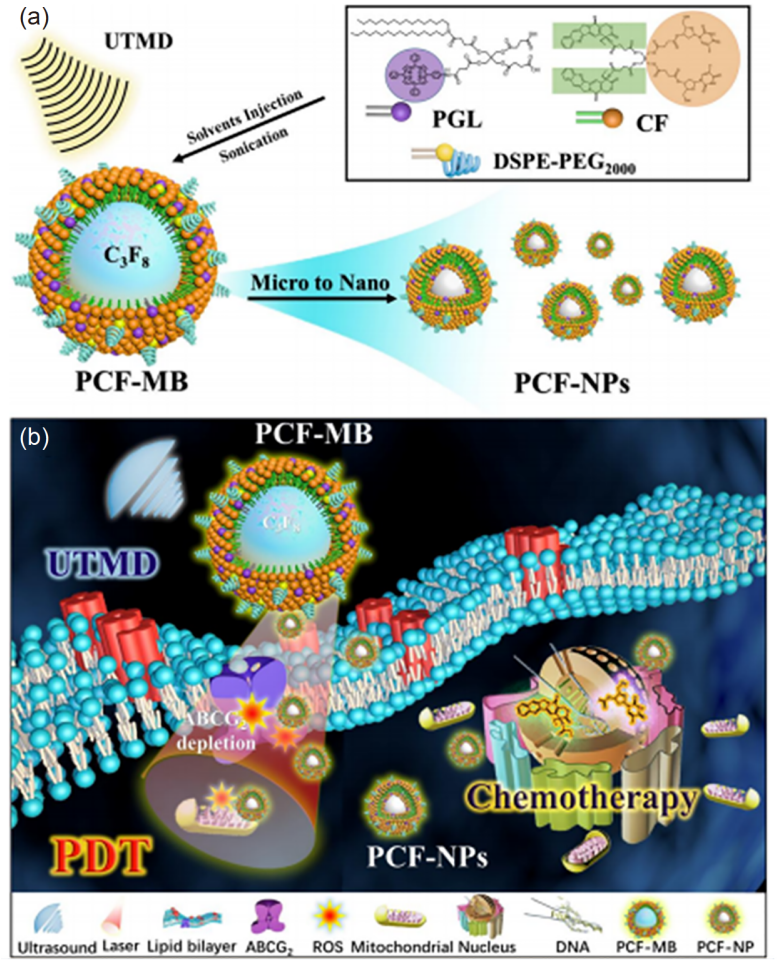

Figure 7 (a) Schematic illustration of self-assembly of PCF-MBs with porphyrin grafted lipid of PGL and camptothecin-floxuridine conjugate of $\mathrm{CF}$, which can be converted into PCF-NPs by ultrasound targeted microbubble destruction. (b) PCF-MBs mediated chemo-photodynamic combination therapy with the assistance of UTMD technique. The therapeutic efficacy may be enhanced since PDT can deplete ABCG2 by which chemo drugs can be pumped out of cancer cells (Reproduced from Ref. [63] with the permission of American Chemical Society Ltd.) (color online).

However, the inherent optical absorbance of biomolecules (e.g., hemoglobin, melanin) often shows background tissue signals to interfere with signals of contrast agents during the in-vivo PAI. To overcome this problem, Liu [67] designed a unique US-responsive photonic probe containing gold nanoparticles (Au NPs) for in-vivo background-free PAI. It could also inspire the design of next-generation US/PA dualmodel imaging agents that allow highly sensitive background-free PAI imaging, which was particularly promising for the accurate PA detection of targets in organs with complicated blood vessels (Figure 8).

\subsubsection{US-NI combination}

Ultrasound waves can map the location of lipid-stabilized MBs after intravenous administration in the body, facilitating an estimate of vascular density and blood flow. MBs with nuclear imaging radioisotopes are generally used to investigate their biodistribution and track their fate and pharmacokinetics [68]. In order to study the biodistribution of US CAs (lipid-shelled MBs), Tartis [69] incorporated the $\left[{ }^{18} \mathrm{~F}\right]$ fluorodipalmitin ( $\left.\left[{ }^{18} \mathrm{~F}\right] \mathrm{FDP}\right)$ into the microbubble shells. PET images suggested that microbubble shell material had a different pharmacokinetic profile from that of freely-injected lipid and liposomal formulations.

Multi-modal imaging specifically enables the study of biological processes, and has great potential for diseasespecific diagnostic evaluation in vivo. Caldahl [70] studied the possibility of targeting adhesion molecules on inflammation-activated endothelial cells and macrophages using polyvinyl alcohol-based MB developed for ultrasound, MRI and NI. These MBs permitted high ligand surface density and allowed the adhension of selective targeting of inflammatory markers such as ICAM-1, VCAM-1 and Eselectin, which offered a chance for diagnosis of inflammation (Figure 9).

\section{US-mediated treatments against fatal diseases}

The selective retention of MBs can be achieved through specific binding to the receptors overexpressed in the diseased regions, and these specific receptors are considered to be specific biomarkers for inflammation, angiogenesis, thrombus formation and so on (Table 2).

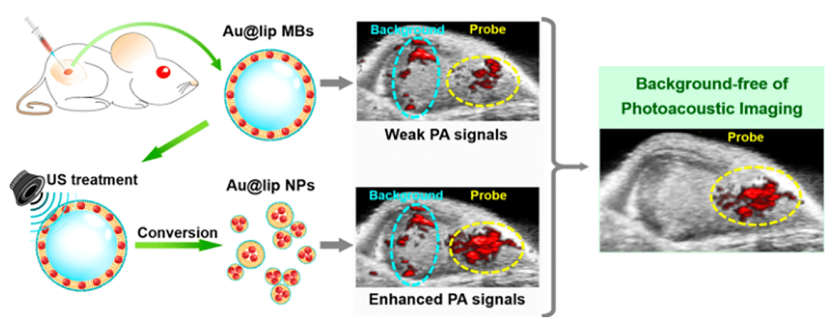

Figure 8 Schematic illustration of US-responsive photonic probes based on MBs containing gold nanoparticles (Au NPs) (Reproduced from Ref. [67] with the permission of American Chemical Society Ltd.) (color online).

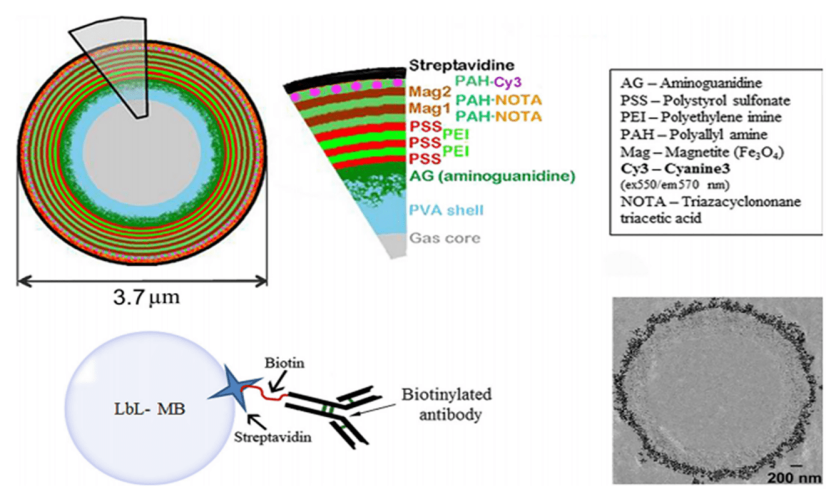

Figure 9 Schematic of MBs with different layers for multi-modal imaging. The layer characteristics of MBs are listed (MB diameter $3.7 \mathrm{~lm}$ in hydrated state as measured by AFM). MBs can be coupled to different antibodies via a streptavidin-biotin linkage (lower left panel). TEM (lower right panel) image of a MB with two layers of SPIONs as used in this study (Reproduced from Ref. [70] with the permission of Springer Ltd.) (color online). 
Table 2 Representative biomarkers for targeted ultrasound treatments

\begin{tabular}{|c|c|c|c|}
\hline Disease & Biomarker & Ligand & Application \\
\hline \multirow{4}{*}{ Inflammation } & E-Selectin & $\begin{array}{l}\text { IELLQAR peptide } \\
\text { GGGIELLQAR peptide }\end{array}$ & $\begin{array}{l}\text { Tumor prognosis } \\
\text { Malignant tumor depiction }\end{array}$ \\
\hline & P-Selectin & Recombinant P-selectin glycoprotein ligand-1 analog & Imaging of transient acute myocardial ischaemia \\
\hline & ICAM-1 $^{\text {a) }}$ & $\begin{array}{l}\text { Monoclonal anti-mouse ICAM-1 } \\
\text { Monoclonal anti-human/mouse ICAM-1 }\end{array}$ & $\begin{array}{l}\text { myocardial ischaemia detection } \\
\text { Targeted delivery for arthrosclerosis }\end{array}$ \\
\hline & VCAM-1 ${ }^{\text {b) }}$ & Monoclonal anti-VCAM-1 & Targeted delivery in mouse aorta model \\
\hline \multirow{2}{*}{ Angiogenesis } & VEGF-2 & $\begin{array}{c}\text { Recombinant VEGF } \\
\text { VEGFR2 }{ }^{\mathrm{c})} \text { antiboby } \\
\text { Biotin-conjugated Monoclonal rat anti-mouse }\end{array}$ & $\begin{array}{l}\text { Tumor angiogenesis imaging } \\
\text { Phase } 0 \text { clinical trial for prostate cancer }\end{array}$ \\
\hline & $\alpha_{\mathrm{v}} \beta_{3}$ & $\begin{array}{l}\text { cRGD pentapeptide } \\
\text { Biotinylated rat Ig antiboby } \\
\text { cRGD peptide }\end{array}$ & $\begin{array}{l}\text { Quantitative visualization of tumor angiogenesis } \\
\text { Detection of early-stage ovarian cancer }\end{array}$ \\
\hline Thrombus & Glycoprotein IIb/IIIa & $\begin{array}{c}\text { cRGD peptide } \\
\text { Thrombin aptamers } \\
\text { Anti-GP IIb/IIIa single-chain antibody }\end{array}$ & $\begin{array}{l}\text { Detection of small clots } \\
\text { Imaging and therapy of thrombolysis }\end{array}$ \\
\hline
\end{tabular}

a) ICAM: Intercellular cell adhesion molecules; b) VCAM: Vascular cell adhesion molecules; c) VEGFR: Vascular endothelial growth factor receptor.

\subsection{Temperature and sonoporation effects}

Among all the ultrasound mechanisms for producing bioeffects, the tissue heating generated is best known and wellunderstood. When the tissue was exposed to ultrasound, the temperature at targeted tissues can be raised to more than $55^{\circ} \mathrm{C}$, which suffices to cause denaturation in proteins leading to coagulative necrosis and cell death [71].

The Magnetic Resonance guided Focused Ultrasound Surgery (MRgFUS) device ExAblate with FDA approval was a perfect example. An MRI and thermal mapping system were included to provide real-time temperature and anatomical monitoring, which offered good guidance in a focused ultrasound beam's destroying the target tissues non-invasively. This provided a good method for the treatment of fibroid and metastatic bone cancer therapy [72]. For the treatment of multiple uterine fibroids, 57 patients were treated with $79 \%$ reported with positive prognosis. The mean reduction in fibroid volume was $13.5 \%$ after 6 months and the non-enhancing volume at approximately $51 \mathrm{~cm}^{3}$ remained during the 6 months after therapy [73]. Moreover, focused ultrasound (FUS) combined with MBs is a feasible, safe and reversible method to enhance BBB permeability [74].

MBs combined with MRI-guided focused ultrasound (MRgFUS) were considered as an effective way to achieve mild hyperthermia at reduced power levels. MRgFUS hyperthermia $\left(42{ }^{\circ} \mathrm{C}\right.$ for $\left.20 \mathrm{~min}\right)$ was used for rabbit thigh muscle or Vx2 tumors, and MBs (Definity, $20 \mu \mathrm{L} / \mathrm{kg}$ ) or saline administered for more than $5 \mathrm{~min}$. To evaluate the effect on drug uptake, liposomal doxorubicin and MBs were injected into rabbit muscle or Vx2 tumor. The hyperthermia of MRgFUS in muscles and tumors resulted in accurate temperature control (mean $=42{ }^{\circ} \mathrm{C}$, root mean square error $\left(\right.$ RMSE) $=0.3{ }^{\circ} \mathrm{C}$ ) (Figure 10). This study has demonstrated the potential application of MBs during mild MRgFUS hyperthermia to reduce the applied power requirements has considerable clinical implications for tumor treatment [75].

US-induced sonoporation is a further application area for US CAs therapeutics. A number of literatures demonstrate that the successful US-mediated imaging and therapy rely on its biophysical effects, such as the thermal effects, radiation force and cavitation. Among these factors, sonoporation resulted from the acoustic cavitation attracts the most attention [76,77]. "Sonoporation" is a transient phenomenon with temporary pores on cell membranes. It can be induced by stable/inertial cavitation and even low-infrequency US without MBs. In the presence of MBs, sonoporation can be enhanced dramatically. Since there is a synergistic effect between US sonoporation and MB-induced shear forces, it makes great contribution to localize drug delivery by increasing blood vessel and/or cell membrane permeability which allows drug molecules transfer through the pores [78].

As shown in Figure 11, exposure to US with different mechanical index (MI) evokes different kinds of cavitation, i) stable or non-inertial cavitation: MBs oscillate steady generating gradient local shear force and acoustic microstreaming; and ii) inertial cavitation: MBs oscillate violently at higher MI until they collapse with localized temperature and pressure increase and free radical generation [79].

\subsection{HIFU-involved treatments against tumors}

High intensity focused ultrasound (HIFU) is being intensively employed in controlled and non-invasive tumor treatments through selective tissue heating. The exposure time is relatively long and continuous in order to get the required high temperature increment for thermal ablation of tumors. The ultimate goal is to induce rapid and irreversible 

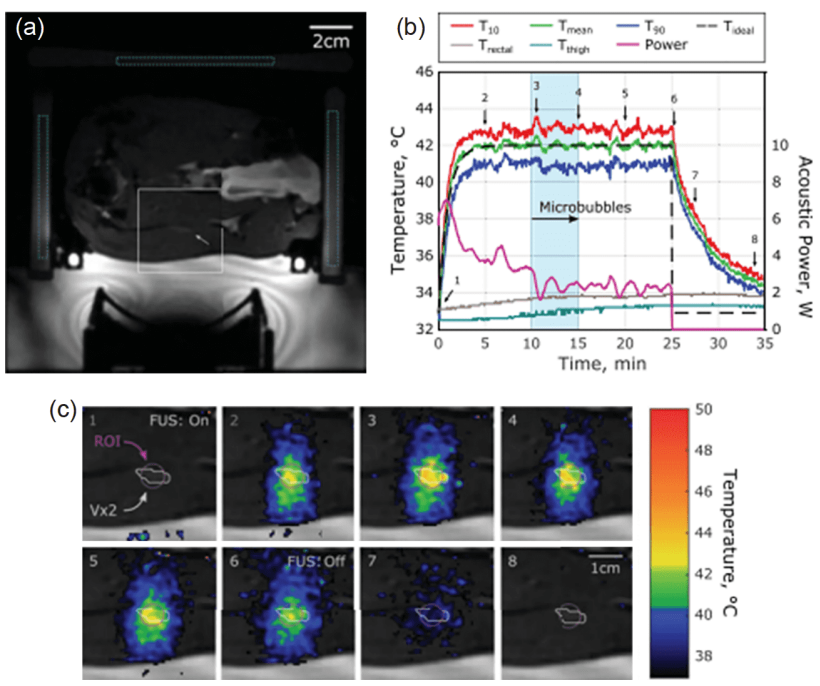

Figure 10 Spatial temperature distribution during hyperthermia. (a) Magnitude image prior to sonication showing rabbit thigh anatomy with an implanted Vx2 tumor (white arrow) and the regions of B0 drift correction regions indicated with the dashed cyan regions. (b) Temperature response during MRgFUS hyperthermia with microbubbles injected at $10 \mathrm{~min}$ into the heating paradigm. (c) Temperature maps in color overlaid upon the magnitude anatomical image in grey (white inset from (a)) at representative time points during heating indicated by the numbers in (b). The $7 \mathrm{~mm}$ circular ROI used for feedback control is shown in purple (Reproduced from Ref. [75] with the permission of Informa Healthcare Ltd.) (color online).

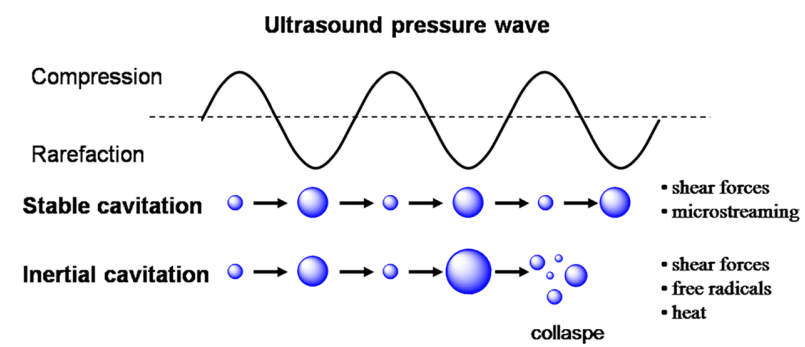

Figure 11 Stable and inertial cavitation of MBs in presence of acoustic pressure waves (color online).

thermal tumor necrosis without noteworthy damage in the surrounding tissue and intervening path [80].

HIFU leading to cell death in targeted tissue enables an advantageous improvement in the non-invasive treatment of localized prostate cancer with fewer side-effects [81]. Lee [82] performed transrectal HIFU therapy on 58 patients, $78 \%$ of who had a decreased prostate-specific antigen (PSA) level to $<0.5 \mathrm{ng} / \mathrm{mL}$ within 3 months. The success rate was closely associated with clinical stage. In conclusion, the utilization of HIFU made great strides in treating prostate cancer in selected patients safely and effectively.

Consisting with the satisfying results in fibroids and prostate cancer, HIFU also has incredibly succeeded in treating breast cancers. Wang [83] prepared nanoscale bubble-generating liposomes containing ammonium bicarbonate [Lip-ABC], which had good stability in vivo and accumu- lated in the tumor interstitial space based on the enhanced permeability and retention effect evaluated by PAI. When applied to synergize HIFU ablation to bovine liver in vitro and implanted breast tumors of BALB/c nude mice, Lip$\mathrm{ABC}$ outperformed the control. Importantly, all mice survived HIFU treatment, suggesting that Lip-ABC is a safe HIFU synergist. Meanwhile, HIFU was proved to effectively relieve pain. Insonation with $1-\mathrm{MHz}$ ultrasound at acoustic intensity greater than $0.5 \mathrm{~W} / \mathrm{cm}^{2}$ improved nerve block effectiveness, increased nerve block reliability, and prolonged both sensory and motor nerve blockade mediated by the hydrophilic ultra-potent local anesthetic, tetrodotoxin [84].

The MBs with HIFU can enhance ablation effects. Bai [85] pretreated with SonoVue and introduced them into rabbits and patients with a solitary uterine fibroid. In the experimental study, the highest temperature and the largest necrotic volume in the liver were observed when HIFU sonication started at 60 seconds after administration of SonoVue. The necrotic volume increased as the SonoVue concentration increased. In the 1-minute SonoVue group of the clinical study, the rate of grey-scale changes was significantly greater than those in other three groups.

The combination of MRI and US has attracted extensive interests since it can realize the real time monitoring of the therapy process [86]. Hence, MRI guidance of the HIFU treatment (MR-HIFU) further accelerated the clinical application with accurate delivery of a high thermal dose to a predefined volume and real-time feedbacks on the well-defined, spatially controlled HIFU heating [87].

The use of MR imaging metrics to predict prostate ablation was not diminished by the presence of silica-shelled ultrasound-triggered phase-shift emulsions (sUPEs). Lesions generated in the presence of sUPEs exhibited more frequent thermal fixation, though there were no significant changes in the ablation areas when comparing arms with and without sUPEs. Thermal fixation corresponded to some qualitative imaging features [88].

Ultrasound can be utilized in synergy with MRI to get dual-modal imaging of tumor for more precise treatment. Based on the advantages of US/MR imaging, Niu [89] has reported a PEGylated magnetite/PFOB co-encapsulated hybrid vesicles for both US/MR imaging and image-guided HIFU ablation. The dual-modal imaging led to accurate diagnosis and monitor of cancer, which could be precisely targeted by MRI-guided HIFU with advantageous improvements in therapeutic efficacy.

Chung [90] evaluated the effect of MBs during HIFU ablation on rabbit liver in vivo. HIFU ablations (intensity of $400 \mathrm{~W} / \mathrm{cm}^{2}$ for $4 \mathrm{~s}$, six times, with a $5 \mathrm{~s}$ interval between exposures) were performed upon 16 in vivo rabbit livers before and after intravenous injection of MBs. Results showed that the volume of coagulative necrosis was significantly larger in the combination MBs and HIFU group 
than in the HIFU alone group $(\mathrm{P}<0.05)$. Also, the time to reach ablation was shorter in the combination of MBs and HIFU group than in the HIFU alone group $(\mathrm{P}<0.05)$. When analyzing the shape of tissue ablation, a pyramidal shape was more prevalently in the HIFU alone group compared to the combination of MBs and HIFU group $(\mathrm{P}<0.05)$. HIFU therapy of in vivo rabbit livers with a microbubble CA produced larger zones of ablation and more cavitary tissue necrosis than without the use of microbubble CAs. Microbubble CAs might be useful in tissue ablation by enhancing the treatment effect of HIFU.

\subsection{Low-intensity ultrasound in therapeutic applica- tions}

The basic principle of using MBs for therapeutic purpose is their ability to enhance vibration-effects generated by US pulses. In 1995, Tachibana [91] observed that the fibrinolysis process could be accelerated in vitro with albumin MBs as diagnostic echo CAs during therapeutic exposure of thrombus to US with urokinase (UK). Xie [92] determined the effectiveness of lipid-encapsulated MBs and US in recanalizing arteriovenous graft thrombi. Clinical studies also demonstrated a synergic effect of US and MBs on sonothrombolysis in patients with arteriovenous dialysis graft thrombosis.

In order to further understand whether MBs could accelerate US-enhanced systemic thrombolysis in stroke patients, Molina [93,94] investigated the parameters of galactosebased MBs to maximize completeness of middle cerebral artery (MCA) recanalization during systemic thrombolysis and continuous $2-\mathrm{Hz}$ pulsed-wave transcranial Doppler (TCD) monitoring. Clinical studies demonstrated that the administration of MBs in combination of tissue plasminogen activator (tPA) could further enhance US-augmented systemic thrombolysis in acute ischemic stroke, leading to a more complete arterial recanalization and to a trend toward better short- and long-term outcomes.

Petit [95] further evaluated the ability of combined US and MBs to degrade the fibrin in the presence or absence of a thrombolytic drug, recombinant tissue plasminogen activator (rtPA). Results demonstrated a strong synergistic effect on fibrin degradation and sonothrombolysis when US, MB, and rtPA were combined. These findings convincingly demonstrated that MBs held significant potential for improving the efficacy of clinically relevant combinations of pharmaceutical and US-based thrombolysis.

Low-intensity US in combination with sensitizing molecules has been widely used in cancer therapy, such as sonodynamic therapy, US-mediated drug delivery and antivascular US therapy. The application of low intensity ultrasound undoubtedly has advantages, namely (i) easy to perform without accuracy need, (ii) relatively inexpensive, (iii) minimal bio-effects in nearby normal cells, (iv) easy targeting to sensitize chemotherapeutic molecules. Even though the exposure time will be prolonged, the repetition and dose control will be easily achieved. The applications examples are introduced thoroughly elsewhere [96].

Gao [97] have studied the sonodynamic therapy (SDT) using 5-aminolevulinic acid (ALA), a precursor of the most commonly used sonosensitizer protoporphyrin IX (PpIX), in combination with low intensity ultrasound. They found that SDT also has an anti-vascular effect by inhibiting in vitro angiogenic processes, including proliferation, migration, invasion and tube formation.

Other mechanisms has been put forward by Saha [98] who described a novel paradigm where the tumor was treated with non-ablative, non-invasive low energy focused ultrasound (LEFU) and a non-toxic low dose 17AAG, an inhibitor of heat-shock protein 90 (HSP90) getting synthetic significant anti-tumor effect in mouse and human prostate cancer (PC) xenografts. Since LEFU caused endoplasmic reticulum (ER) stress and unfolded protein response (UPR) in tumor cells, whilst treatment of 17AAG further accelerated the apoptosis and tumor growth inhibition.

\subsection{US-mediated controllable drug/gene delivery}

With the development of molecular imaging, the utilization of MBs as targeted delivery vehicles is one of the most intensively researched applications of US CAs. Considering that MBs are outstanding carriers to efficiently load hydropholic/hydrophilic drugs and genes, the therapeutic payloads can be controllably released at the diseased lesions upon exposing with an ultrasonic field [99].

The promising novel acoustic cluster therapy (ACT) has caught increasingly attention. Its particularity lies in the cluster dispersion mixing negatively charged MBs with postively charged microdroplets. A dispersion of clusters is co-injected intravenously with a drug followed by local, low power insonation with regular medical imaging US. A large bubble $(20-30 \mu \mathrm{m})$ will be generated in-situ, which transiently deposits in the microvasculature leading to occluding blood flow for 5-10 min. This further enhances the local drug efficacy since a rapid wash out of drug will be prevented [100,101]. Van Wamel's group [102] combined ACT with abraxane and paclitaxel for the treatment of human prostate adenocarcinoma in mouse model, which induced a 10 -fold reduction in tumor growth rate and complete remission $>40 \%$ in animals.

Although drug-microbubble co-administration has been confirmed to increase permeability/transfection efficiency of drugs/genes in targeted tissues, in-situ incorporations of drugs/genes into MBs become more attractive because that the payloads can be transported locally through blood walls and this process can be precisely monitored by US imaging 
$[103,104]$. There are a myriad of techniques to incorporate drugs/genes into the MBs chemically or physically (Figure 12) $[105,106]$.

After the intravenous injection into the body, MBs will be freely circulating in the blood vessels, and their sizes inhibit their extravasation out of the vasculature. However, once MBs are subjected to an extrinsic US field with an appropriate intensity, they will be disintegrated and give rise to a series of phenomena. MBs will further burst into pieces while releasing the loaded drugs/genes. In the meantime, immediate rupture of micro-vessels will come into existence and the vascular permeability will be enhanced, which may overcome the compact endothelial cell barriers into the tumor area. As the tumor cells located in proximity are relaxed by sonoporation, the permeability between cell membranes is dramatically boosted. Therefore, the released drugs/genes may efficiently penetrate through the vascular intervals and transient gaps on the cell membranes, reach the targeted tissues and exhibit their therapeutic performances.

Besides showing potential for facilitating the delivery of standard drugs to target cells, the sonoporation has also been shown to hold significant potential for improving the delivery and the efficacy of genetic interventions based on plasmid DNA, small interfering RNA, etc.

US-mediated gene delivery is a minimally invasive method for gene therapy with modest gene transfection efficiency, but there is a high organ and tissue specificity. A pioneering proof-of-principle study in this field has been reported by Leong-Poi [107], who introduced a noninvasive method for gene delivery by using US-mediated destruction of intravenously administered DNA-bearing carrier MBs. Cationic lipid shelled MBs were coupled to complex plasmid DNA encoding for VEGF for inducing therapeutic arteriogenesis in chronically ischemic skeletal muscle. Their finding showed that these gene-loaded MBs were able to enhance the expression of VEGF in ischemic hind limbs, to increase microvessel density, and thereby to improve microvascular blood flow.

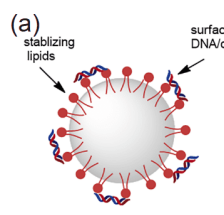

(d)

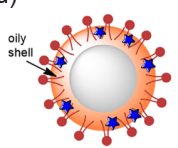

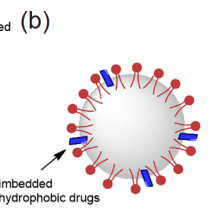

(e)

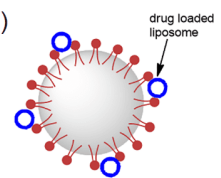

(c)

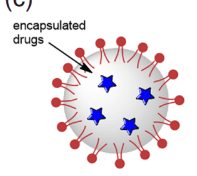

(f)

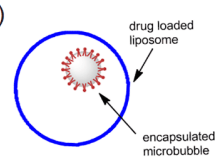

Figure 12 Versatile techniques to incorporate drugs/genes with MBs. (a) Surface attachment. (b) Shell imbedding. (c) Cone encapsulation. (d) Oily shell thickening. (e) Lipsome attachment. (f) MBs encapsulation into liposome (Reproduced from Ref. [106] with the permission of Dovepress Ltd.) (color online).
Dewitte [108] also reported mRNA loaded MBs for the US-triggered transfection of dendritic cell (DCs). Antigen mRNA and TriMix mRNA were bound to the lipid MBs and their transfection efficiency was evaluated with US irradiation. Once the MBs and the mRNA complex were localized within the lymph nodes, higher intensity US pulses could induce localized MBs implosion, resulting in spatiotemporally improved delivery of both antigen mRNA and immunomodulating TriMix to intranodal DCs. The maturated antigen-presenting dendritic cells then led to a strong reduction of tumor volume.

Meanwhile, surface-engineered NBs also have a wider application scope. Li [109] designed "all-in-one" NBs using electrostatic adsorption and further filled them with gas after lyophilization. These NBs could respond to $\mathrm{pH}$-/light-sensitive drug-release stimuli, and were ultimately used for tumor-targeted NIR/MR/US imaging and combined chemophotothermal therapy.

\subsection{Endoscopic ultrasound therapy}

Endoscopic ultrasonography (EUS) is a digestive examination technique that combines endoscopy and ultrasound. A miniature high-frequency ultrasound transducer is placed on the top of an endoscope. When the endoscope is inserted into the body cavity, the gastrointestinal mucosal lesions are directly visualized by the endoscope, and so the real-time scanning by the endoscopic ultrasound can be used to detect the histological features and the US images of the surrounding organs so as to improve the diagnostic efficiency. The applications of EUS include: i) the judgement of the invasion depth, lymph node metastasis and possibility of surgical resection of gastrointestinal tumors; ii) the determination of the origin and nature of submucosal tumors of the digestive; iii) the diagnosis and staging of duodenal papillary and ampullary carcinoma; iv) the diagnosis of benign and malignant lesions in the pancreas, gallbladder and common bile duct; and v) the diagnosis of benign and malignant colorectal lesions.

The pancreas cancers have a poorest prognosis among all cancers, as many tumors were unable to be detected until found in surgery. Surgical viability typically relies on endoscopic ultrasound. Jiang [110] described the development of a dual-frequency piezoelectric transducer for rotational endoscopic imaging and the visualization of MBs-specific superharmonic signals. It may enable direct visualization of small vessels from within the lumen of larger vessels such as the portal vein or superior mesenteric vein (Figure 13).

\section{Challenges and perspectives}

The emerging innovations on functional micro/nanobubbles 

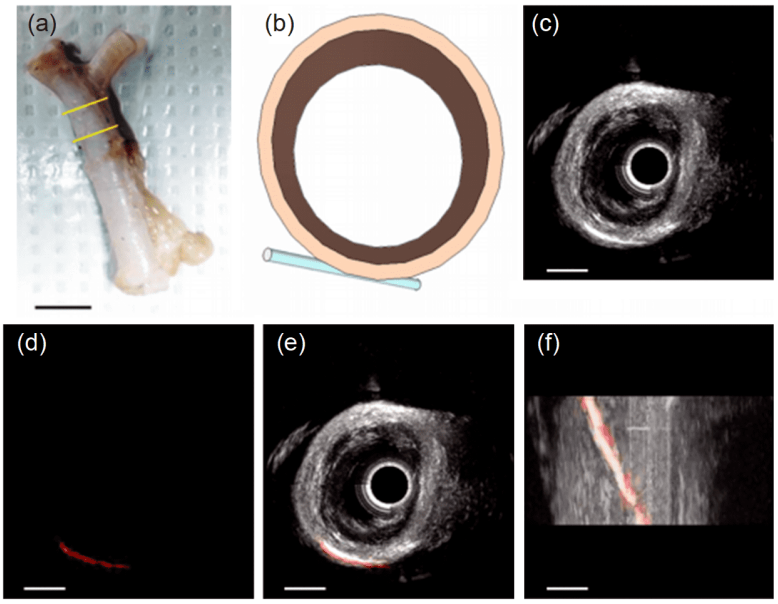

Figure 13 (a) Ex vivo porcine splenic artery specimen used for imaging experiments, with the imaged section indicated by yellow bars. Scale bar represents $1 \mathrm{~cm}$. (b) Schematic of ex vivo artery section and cellulose tube filled with MBs for imaging experiment. (c) 3D rendering of $\mathrm{B}$ mode $(20 \mathrm{MHz})$ image acquired in arterial section (Scale bar $=2 \mathrm{~mm}$ ). (d) $3 \mathrm{D}$ rendering of dual-frequency (transmit $4 \mathrm{MHz}$, receive $20 \mathrm{MHz}$ ) image of MBs in tube acquired in arterial section. Combined 3D rendering of Bmode (grayscale) and dual-frequency (red) imaging volumes shows contrast-specific signal originates only from MBs within the tube in (e) short and (f) long-axis views (Scale bar $=2 \mathrm{~mm}$ ).(Reproduced from Ref. [110] with the permission of Medscape Ltd.) (color online).

provide an unprecedented opportunity for non-invasively monitoring of the biological and pathological processes at a molecular and cellular level. The online detection and realtime analysis not only supply the theranostic methodology with high precision and customization, but also give every access to individualized medicine. On the other hand, the prominent properties of US and advanced US-based technologies have boosted the development of new theranostic strategies for various diseases at early stage (Figure 14). Yet, the efficient association between functional biomaterials and US-based techniques is still in its infancy, and tremendous investigations are required to be completed. For example, the methods on how to realize the intuitive visualization and quantitative evaluation are scarce so far. And these methods should be capable to monitor the entire processes of disease occurrence, development and metastasis in a relatively longterm manner. In terms of theranostic stages with functional micro/nano bubbles, the visualization and quantitative evaluation should always include three main steps as the-abovementioned: 1) the specific biomarker-targeted anchoring of MBs within the vasculature; 2) the controllable release of therapeutic drugs or secondary diagnostic agents out of MBs and their extravasation into diseased tissues as a result of sonoporation/cavitation effect; 3 ) secondary or multi-stage targeting of functional agents into diseased areas for treatment purposes. As discussed above, although the combinations of US and other modalities such as fluorescence, MRI and photoacoustic imaging have shown great promises to translational research, more elaborate incorporations among

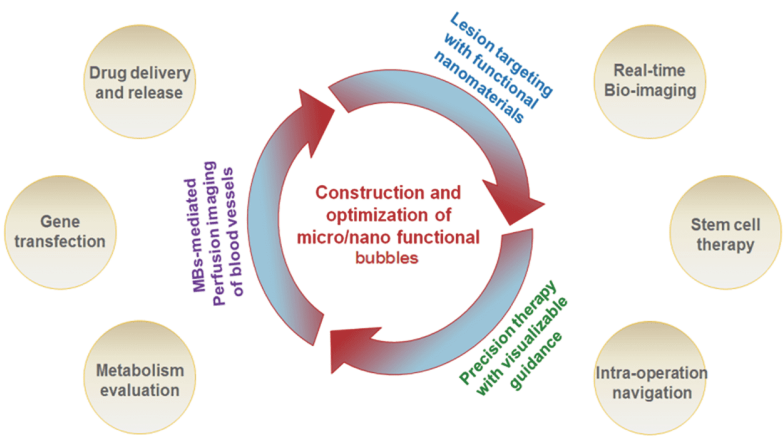

Figure 14 Functional micro/nano bubbles in US-mediated theranostics for precision therapy with visualizable guidance (color online).

versatile theranostic modalities may be introduced to further improve the sensitivity and quantitativeness of the evaluations.

Another issue with an urgent need to handle is the development of novel micro/nano bubbles as next-generation multifunctional carriers to match the US instrumentations. To this end, it is a major challenge on how to elaborately modulate the size, in vivo stability, drug encapsulation of micro/nanobubbles and optimize ultrasonic parameters to acquire the best stimuli efficiency for consecutive drug release or therapy processes. Meanwhile, the bio-safety and translational possibility to clinics should be very carefully pondered. A feasible way is to fabricate size-tunable carriers with FDA-approved biomaterials, and by segmenting with diverse size distribution, these biomaterials can find versatile biomedical applications and enhance their bioavailability. Micro-sized biomaterials are capable to target the specific biomarkers within the blood vessels, and nano-sized droplets or particles are qualified to penetrate endothelial cells for intracellular therapy. Therefore, multiple roles are endorsed to this hybrid scaffold for a stimuli-to-respond theranostic style, and each composition unit will smartly respond to ambient changes either internal micro-environmental conditions or external US deposition.

The outbreak of Coronavirus (COVID-19) has caused an atypical acute respiratory pandemic disease all over the globe in 2019, and severe shunt in patients with atypical acute respiratory distress syndrome can be detected and characterized by a handheld ultrasound device using a saline microbubble contrast agent [111]. The use of contrast enhanced ultrasound can assess the changes in the lungs of the pleural appendages in COVID-19 patients, which utilizes SF6-loaded microbubbles to digital storage [112]. Microbubbles can stick to the damaged vessels endothelium, and by combining different drugs, targeted therapy can be achieved with low toxic and side effects. Perfluorobutane microbubbles with a cell membrane fluidizer are able to load pro-inflammatory cytokine (TNF- $\alpha$ or IL-6) inhibitors to achieve reduction of related symptoms with little damage to blood vessels $[113,114]$. 
In summary, functional micro/nano bubbles in association with US-mediated theranostic techniques have shed a bright future in the trans-disciplinary fields of functional agents, bioengineering, ultrasonography and translational medicine. As an emerging subject and research area, few publications have been made so far and there exist attractive chances along with bottleneck problems at the same time. Nevertheless, with all the accumulating achievements and evergrowing developments, more elaborately designed bubbles and broader applications related to the US-mediated theranostics will be anticipated to come into use, and successful from-bench-to-bedside translations may be realized in the near future to better meet the demands of human health and disease treatment.

Acknowledgements This research was financially supported by the National Natural Science Foundation of China (21575106, 82072057).

Conflict of interest The authors declare no conflict of interest.

1 (a)American National Standard Institute. American national standard: accoustical terminology. New York: Acoustical Society of America.; (b)1994.

2 Oda F, Corso JF. J Acoust Soc Am, 1962, 34: 746

3 Liu HL, Fan CH, Ting CY, Yeh CK. Theranostics, 2014, 4: 432-444

4 Unger E, Porter T, Lindner J, Grayburn P. Adv Drug Deliver Rev, 2014, 72: 110-126

5 Sun RR, Noble ML, Sun SS, Song S, Miao CH. J Control Release, 2014, 182: 111-120

6 Wang X, Niu D, Li P, Wu Q, Bo X, Liu B, Bao S, Su T, Xu H, Wang Q. ACS Nano, 2015, 9: 5646-5656

7 Leon A, Perera R, Nittayacharn P, Cooley M, Jung O, Exner AA. $A d v$ Cancer Res, 2018, 139: 57-84

8 Gramiak R, Shah PM. Invest Rad, 1968, 3: 356-366

9 Kremkau FW, Gramiak R, Carstensen EL, Shah PM, Kramer DH. Am J Roentgenology, 1970, 110: 177-183

10 Feinstein SB, Cheirif J, Ten Cate FJ, Silverman PR, Heidenreich PA, Dick C, Desir RM, Armstrong WF, Quinones MA, Shah PM. J Am College Cardiology, 1990, 16: 316-324

11 Appis AW, Tracy MJ, Feinstein SB. Echo Res Practice, 2015, 2: R55-R62

12 Keller MW, Glasheen W, Kaul S. J Am Soc Echocard, 1989, 2: 4852

13 Podell S, Burrascano C, Gaal M, Golec B, Maniquis J, Mehlhaff P. Biotechnol Appl Biochem, 1999, 30: 213-223

14 Goertz DE, de Jong N, van der Steen AFW. Ultrasound Med Biol, 2007, 33: 1376-1388

15 Schneider M. Eur Radiol, 1999, 9: S347-S348

16 Sontum PC. Ultrasound Med Biol, 2008, 34: 824-833

17 Bhutani MS, Hoffman BJ, van Velse A, Hawes RH. Endoscopy, 1997, 29: 635-639

18 Christiansen C, Kryvi H, Sontum PC, Skotland T. Biotechnol Appl Biochem, 1994, 19: 307-320

19 Keller MW, Feinstein SB, Watson DD. Am Heart J, 1987, 114: 570575

20 Cohen JL, Cheirif J, Segar DS, Gillam LD, Gottdiener JS, Hausnerova E, Bruns DE. J Am College Cardiology, 1998, 32: 746-752

21 Cavalieri F, Ashokkumar M, Grieser F, Caruso F. Langmuir, 2008, 24: $10078-10083$

22 Wang S, Hossack JA, Klibanov AL. J Drug Targeting, 2018, 26: 420-434

23 Wheatley M. Biomaterials, 1990, 11: 713-717
24 Narayan P, Wheatley MA. Polym Eng Sci, 1999, 39: 2242-2255

25 Liu W, Wen S, Jiang L, An X, Zhang M, Wang H, Zhang Z, Zhang G, Shi X. CNANO, 2014, 10: 543-552

26 Lukianova-Hleb EY, Ren X, Zasadzinski JA, Wu X, Lapotko DO. Adv Mater, 2012, 24: 3831-3837

27 Shen Y, Lv W, Yang H, Cai W, Zhao P, Zhang L, Zhang J, Yuan L, Duan Y. Cancer Lett, 2019, 455: 14-25

28 Bauer A, Blomley M, Leen E, Cosgrove D, Schlief R. Eur Radiol, 1999, 9: S349-S352

29 Forsberg F, Goldberg BB, Liu JB, Merton DA, Rawool NM, Shi WT. Radiology, 1999, 210: 125-132

30 Fokong S, Siepmann M, Liu Z, Schmitz G, Kiessling F, Gätjens J. Ultrasound Med Biol, 2011, 37: 1622-1634

31 Koczera P, Wu Z, Fokong S, Theek B, Appold L, Jorge S, Möckel D, Liu Z, Curaj A, Storm G, van Zandvoort M, Kiessling F, Lammers T. Drug Deliv Transl Res, 2012, 2: 56-64

32 Liu Z, Lammers T, Ehling J, Fokong S, Bornemann J, Kiessling F, Gätjens J. Biomaterials, 2011, 32: 6155-6163

33 Cavalieri F, El Hamassi A, Chiessi E, Paradossi G. Langmuir, 2005, 21: 8758-8764

34 Brismar TB, Grishenkov D, Gustafsson B, Härmark J, Barrefelt A, Kothapalli SVVN, Margheritelli S, Oddo L, Caidahl K, Hebert H, Paradossi G. Biomacromolecules, 2012, 13: 1390-1399

35 Böhmer MR, Schroeders R, Steenbakkers JAM, de Winter SHPM, Duineveld PA, Lub J, Nijssen WPM, Pikkemaat JA, Stapert HR. Colloids Surfs A-Physicochem Eng Aspects, 2006, 289: 96-104

36 Unger EC, Porter T, Culp W, Labell R, Matsunaga T, Zutshi R. Adv Drug Deliver Rev, 2004, 56: 1291-1314

37 Sanchez K, Barr RG. Ultrasound Q, 2009, 25: 171-173

38 Jiang N, Xie B, Zhang X, He M, Li K, Bai J, Wang Z, He J, Zhang L. Cardiovasc Intervent Radiol, 2014, 37: 1321-1328

39 Singhal S, Moser CC, Wheatley MA. Langmuir, 1993, 9: 2426-2429

40 Sirlin CB, Girard MS, Steinbach GC, Baker KG, Broderdorf SK, Hall LA, Mattrey RF. Int J Imag Syst Technol, 2015, 8: 82-88

41 Pelura TJ. Academic Rad, 1998, 5: S69-S71

42 Ota T, Ono S. BJR, 2004, 77: 148-149

43 Wu Y, Peng H, Zhao X. Ultrasound Med Biol, 2015, 41: 967-974

44 Oeffinger BE, Wheatley MA. Ultrasonics, 2004, 42: 343-347

45 Xu JS, Huang J, Qin R, Hinkle GH, Povoski SP, Martin EW, Xu RX. Biomaterials, 2010, 31: 1716-1722

46 Son S, Min HS, You DG, Kim BS, Kwon IC. Nano Today, 2014, 9: $525-540$

47 Xing Z, Wang J, Ke H, Zhao B, Yue X, Dai Z, Liu J. Nanotechnology, 2010, 21: 145607

48 Zheng R, Yin T, Wang P, Zheng R, Zheng B, Cheng D, Zhang X, Shuai XT. IJN, 2012, 895

49 Liberman A, Martinez HP, Ta CN, Barback CV, Mattrey RF, Kono Y, Blair SL, Trogler WC, Kummel AC, Wu Z. Biomaterials, 2012, 33: 5124-5129

50 Hundley WG, Kizilbash AM, Afridi I, Franco F, Peshock RM, Grayburn PA. J Am College Cardiology, 1998, 32: 1426-1432

51 Rainbird AJ, Mulvagh SL, Oh JK, McCully RB, Klarich KW, Shub C, Mahoney DW, Pellikka PA. J Am Soc Echocard, 2001, 14: 378385

52 Darge K, Zieger B, Rohrschneider W, Ghods S, Wunsch R, Troeger J. Am J Roentgenology, 2012, 177: 1411-1415

53 Ferrante EA, Pickard JE, Rychak J, Klibanov A, Ley K. J Control Release, 2009, 140: 100-107

54 Quillard T, Libby P. Circ Res, 2012, 111: 231-244

55 Schreglmann SR, Bauer R, Hägele-Link S, Bhatia KP, Natchev P, Wegener N, Lebeda A, Werner B, Martin E, Kägi G. Neurology, 2017, 88: 1329-1333

56 Jolesz FA. Апnи Rev Med, 2009, 60: 417-430

57 Lammers T, Koczera P, Fokong S, Gremse F, Ehling J, Vogt M, Pich A, Storm G, van Zandvoort M, Kiessling F. Adv Funct Mater, 2015, 25: $36-43$

58 Xiao YD, Paudel R, Liu J, Ma C, Zhang ZS, Zhou SK. Int J Mol 
Med, 2016, 38: 1319-1326

59 Feshitan JA, Vlachos F, Sirsi SR, Konofagou EE, Borden MA. Biomaterials, 2012, 33: 247-255

60 Feshitan JA, Boss MA, Borden MA. Langmuir, 2012, 28: 1533615343

61 Liu Z, Koczera P, Doleschel D, Kiessling F, Gätjens J. Chem Commun, 2012, 48: 5142-5144

62 Liu Z, Shi C, Li Y, Song Y, Xu Q. RSC Adv, 2016, 6: 32710-32714

63 Chen M, Liang X, Gao C, Zhao R, Zhang N, Wang S, Chen W, Zhao B, Wang J, Dai Z. ACS Nano, 2018, 12: 7312-7326

64 Pellow C, O'Reilly MA, Hynynen K, Zheng G, Goertz DE. Nano Lett, 2020, 20: 4512-4519

65 Zackrisson S, van de Ven SMWY, Gambhir SS. Cancer Res, 2014, 74: 979-1004

66 Ishihara. J Anal Oncol, 2013, 2: 81

67 Meng Z, Zhou X, She J, Zhang Y, Feng L, Liu Z. Nano Lett, 2019, 19: 8109-8117

68 Ferrara KW, Borden MA, Zhang H. Acc Chem Res, 2009, 42: 881892

69 Tartis MS, Kruse DE, Zheng H, Zhang H, Kheirolomoom A, Marik J, Ferrara KW. J Control Release, 2008, 131: 160-166

70 Ahmed M, Gustafsson B, Aldi S, Dusart P, Egri G, Butler LM, Bone D, Dähne L, Hedin U, Caidahl K. Cel Mol Bioeng, 2019, 12: 15-32

71 Subramaniam R, Omar S, Madenan N, Abdullah B. Ultrasound Obst Gyn, 2013,10: 11-17

72 Ringold S. J Am Med Ass, 2004, 292: 2826

73 Hindley J, Gedroyc WM, Regan L, Stewart E, Tempany C, Hynnen K, Macdanold N, Inbar Y, Itzchak Y, Rabinovici J, Kim K, Geschwind JF, Hesley G, Gostout B, Ehrenstein T, Hengst S, Sklair-Levy M, Shushan A, Jolesz F. Am J Roentgenology, 2012, 183: 1713-1719

74 Arif WM, Elsinga PH, Gasca-Salas C, Versluis M, Martínez-Fernández R, Dierckx RAJO, Borra RJH, Luurtsema G. J Control Release, 2020, 324: 303-316

75 Santos MA, Wu SK, Li Z, Goertz DE, Hynynen K. Int $J$ Hyperthermia, 2018, 35: 599-611

76 Zhao R, Liang X, Zhao B, Chen M, Liu R, Sun S, Yue X, Wang S. Biomaterials, 2018, 173: 58-70

77 Li WP, Su CH, Chang YC, Lin YJ, Yeh CS. ACS Nano, 2016, 10: 2017-2027

78 Meng L, Liu X, Wang Y, Zhang W, Zhou W, Cai F, Li F, Wu J, Xu L, Niu L, Zheng H. Adv Sci, 2019, 6: 1900557

79 Lentacker I, De Cock I, Deckers R, De Smedt SC, Moonen CTW. Adv Drug Deliver Rev, 2014, 72: 49-64

80 van den Bijgaart RJE, Eikelenboom DC, Hoogenboom M, Fütterer JJ, den Brok MH, Adema GJ. Cancer Immunol Immunother, 2017, 66: $247-258$

81 Uchida T, Nakano M, Hongo S, Shoji S, Nagata Y, Satoh T, Baba S, Usui Y, Terachi T. Int J Urology, 2012, 19: 187-201

82 Lee HM, Hong JH, Choi HY. Prostate Cancer Prostatic Dis, 2006, 9: 439-443

83 Feng G, Hao L, Xu C, Ran H, Zheng Y, Li P, Cao Y, Wang Q, Xia J, Wang Z. IJN, 2017, Volume 12: 4647-4659

84 Cullion K, Petishnok LC, Sun T, Santamaria CM, Pemberton GL, McDannold NJ, Kohane DS. Drug Deliv Transl Res, 2020, 10: 15071516

85 Cheng C, Xiao Z, Huang G, Zhang L, Bai J. BJOG-Int J Obstet Gy, 2017, 124: $78-86$
86 Liu Z, Kiessling F, Gätjens J. J Nanomaterials, 2010, 2010: 894303

87 Hijnen N, Langereis S, Grüll H. Adv Drug Deliver Rev, 2014, 72: 65-81

88 Anthony GJ, Bader KB, Wang J, Zamora M, Ostdiek A, Antic T, Krueger S, Weiss S, Trogler WC, Blair SL, Kummel AC, Sammet S. Med Phys, 2019, 46: 774-788

89 Niu D, Wang X, Li Y, Zheng Y, Li F, Chen H, Gu J, Zhao W, Shi J. Adv Mater, 2013, 25: 2686-2692

90 Chung DJ, Cho SH, Lee JM, Hahn ST. Eur J Rad, 2012, 81: e519e523

91 Tachibana K, Tachibana S. Circulation, 1995, 92: 1148-1150

92 Xie F, Tsutsui JM, Lof J, Unger EC, Johanning J, Culp WC, Matsunaga T, Porter TR. Ultrasound Med Biol, 2005, 31: 979-985

93 Molina CA, Ribo M, Rubiera M, Montaner J, Santamarina E, Delgado-Mederos R, Arenillas JF, Huertas R, Purroy F, Delgado P, Alvarez-Sabin J. Stroke, 2006, 37: 425-429

94 Molina CA, Barreto AD, Tsivgoulis G, Sierzenski P, Malkoff MD, Rubiera M, Gonzales N, Mikulik R, Pate G, Ostrem J, Singleton W, Manvelian G, Unger EC, Grotta JC, Schellinger PD, Alexandrov AV. Ann Neurol, 2009, 66: 28-38

95 Petit B, Yan F, Bussat P, Bohren Y, Gaud E, Fontana P, Tranquart F, Allémann E. J Drug Deliver Sci Tech, 2015, 25: 29-35

96 Wood AKW, Sehgal CM. Ultrasound Med Biol, 2015, 41: 905-928

97 Gao Z, Zheng J, Yang B, Wang Z, Fan H, Lv Y, Li H, Jia L, Cao W. Cancer Lett, 2013, 335: 93-99

98 Saha S, Bhanja P, Partanen A, Zhang W, Liu L, Tomé WA, Guha C. Oncoscience, 2014, 1: 434-445

99 Liu Y, Miyoshi H, Nakamura M. J Control Release, 2006, 114: 8999

100 Sontum P, Kvåle S, Healey AJ, Skurtveit R, Watanabe R, Matsumura M, Østensen J. Int J Pharm, 2015, 495: 1019-1027

101 Park K. J Control Release, 2016, 236: 117

102 van Wamel A, Sontum PC, Healey A, Kvåle S, Bush N, Bamber J, de Lange Davies C. J Control Release, 2016, 236: 15-21

103 Fan Z, Kumon RE, Deng CX. Therapeutic Deliver, 2014, 5: 467-486

104 Fan Z, Chen D, Deng CX. J Control Release, 2013, 170: 401-413

105 Unger EC, Matsunaga TO, McCreery T, Schumann P, Sweitzer R, Quigley R. Eur J Rad, 2001, 42: 160-168

106 Ibsen S, Schutt S, Esener S. DDDT, 2013, 7: 375-388

107 Leong-Poi H, Kuliszewski MA, Lekas M, Sibbald M, Teichert-Kuliszewska K, Klibanov AL, Stewart DJ, Lindner JR. Circ Res, 2007, 101: $295-303$

108 Dewitte H, Van Lint S, Heirman C, Thielemans K, De Smedt SC, Breckpot K, Lentacker I. J Control Release, 2014, 194: 28-36

109 Li T, Zhou J, Zhang C, Zhi X, Niu J, Fu H, Song J, Cui D. NPG Asia Mater, 2018, 10: 1046-1060

110 Lindsey BD, Kim J, Dayton PA, Jiang X. IEEE Trans Ultrason Ferroelect Freq Contr, 2017, 64: 1078-1086

111 Rajendram R, Hussain A, Mahmood N, Kharal M. Ultrasound J, 2020, 12: 49

112 Jung EM, Stroszczynski C, Jung F. CH, 2020, 75: 19-26

113 Tsutsui JM, Xie F, Cano M, Chomas J, Phillips P, Radio SJ, Lof J, Porter TR. J Am College Cardiology, 2004, 44: 1036-1046

114 Porter TR, Mulvagh SL, Abdelmoneim SS, Becher H, Belcik JT, Bierig M, Choy J, Gaibazzi N, Gillam LD, Janardhanan R, Kutty S, Leong-Poi H, Lindner JR, Main ML, Mathias Jr. W, Park MM, Senior R, Villanueva F. J Am Soc Echocard, 2018, 31: 241-274 